\title{
Gemeinsam gegen Krebs
}

\section{Adrian Ritter}

Freier Journalist

Der 2. Schweizerische Krebskongress widmete sich der "Qualität in der Onkologie». Weil Krebserkrankungen zunehmen und immer öfter chronisch verlaufen, wird die interdisziplinäre Zusammenarbeit umso wichtiger, lautet das Fazit.

Was ist Qualität? Und was macht gute Qualität in der Behandlung von Krebskranken aus? Mit diesen Fragen beschäftigte sich im August der 2. Schweizer Krebskongress an der Universität Fribourg. In ihren einführenden Referaten näherten sich der emeritierte Philosophieprofessor Georg Kohler und Hans Stalder, ehemaliger Chefarzt der Medizinischen Poliklinik und des Département de Médecine communautaire am Universitätsspital Genf, den Fragen von der philosophischen beziehungsweise ärztlichen Seite an .

Qualitäten beziehen sich immer auf menschliche Wahrnehmungen, sagte Georg Kohler. Zwar werde versucht, Qualität als Quantität zugänglich zu machen sie bleibe dabei aber "Quasiqualität». Letztlich sei Qualität nur über die menschliche Erfahrung zugänglich. Entsprechend bleibe jenseits aller messbaren Parameter die Frage an die Patientinnen und Patienten sinnvoll: «Fühlen Sie sich krank?» Oder: «Fühlen Sie sich gesund?»

\section{Schweizerischer Krebskongress}

Der Schweizerische Krebskongress fand erstmals 2014 statt, initiiert von den Leistungserbringern in der Krebsbehandlung. In diesem Jahr wurde er gemeinsam mit Akteuren der Nationalen Strategie gegen Krebs (NSK) durchgeführt.

Der Anlass versteht sich als Think Tank, jährliches Arbeitstreffen und Forum zur kontinuierlichen Verbesserung von Vorsorge, Behandlung, Betreuung und Forschung in der Onkologie. Die Veranstaltung soll die Vernetzung der verschiedenen Leistungserbringer und Organisationen fördern. Die Präsentationen zum diesjährigen Krebskongress sind zu finden unter www.swisscancercongress.ch

\section{Weniger ist mehr}

Hans Stalder formulierte es provokativer: «Man sollte sich nie auf Guidelines beziehen!» Der einzelne Patient befinde sich selten in der Mitte der Normalverteilung, auf welche die Guidelines sich beziehen. Zudem wider-

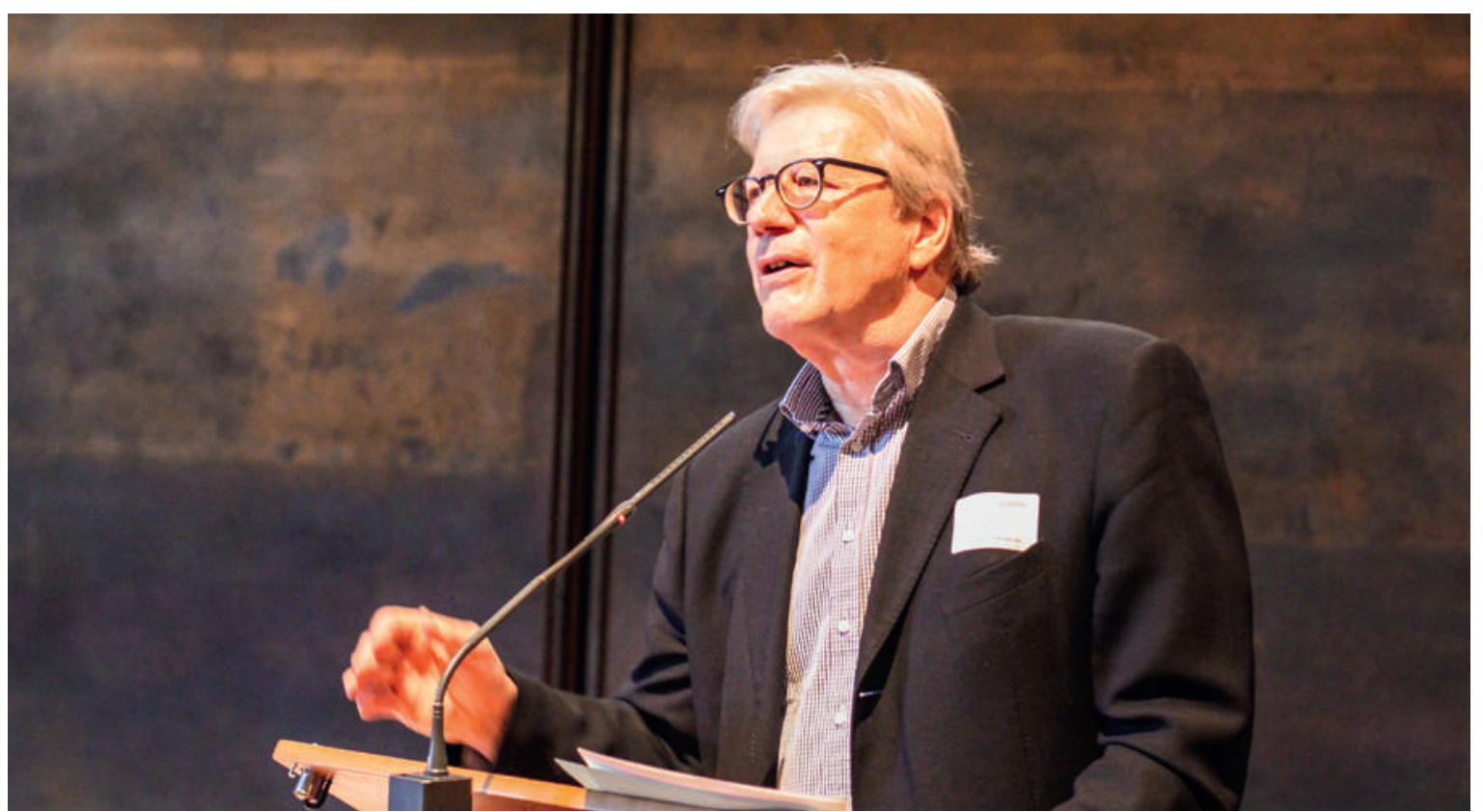

Qualität ist jenseits von Messbarkeit, sie ist eine Frage der persönlichen Wahrnehmung, gibt der emeritierte Philosophieprofessor Georg Kohler zu bedenken. 


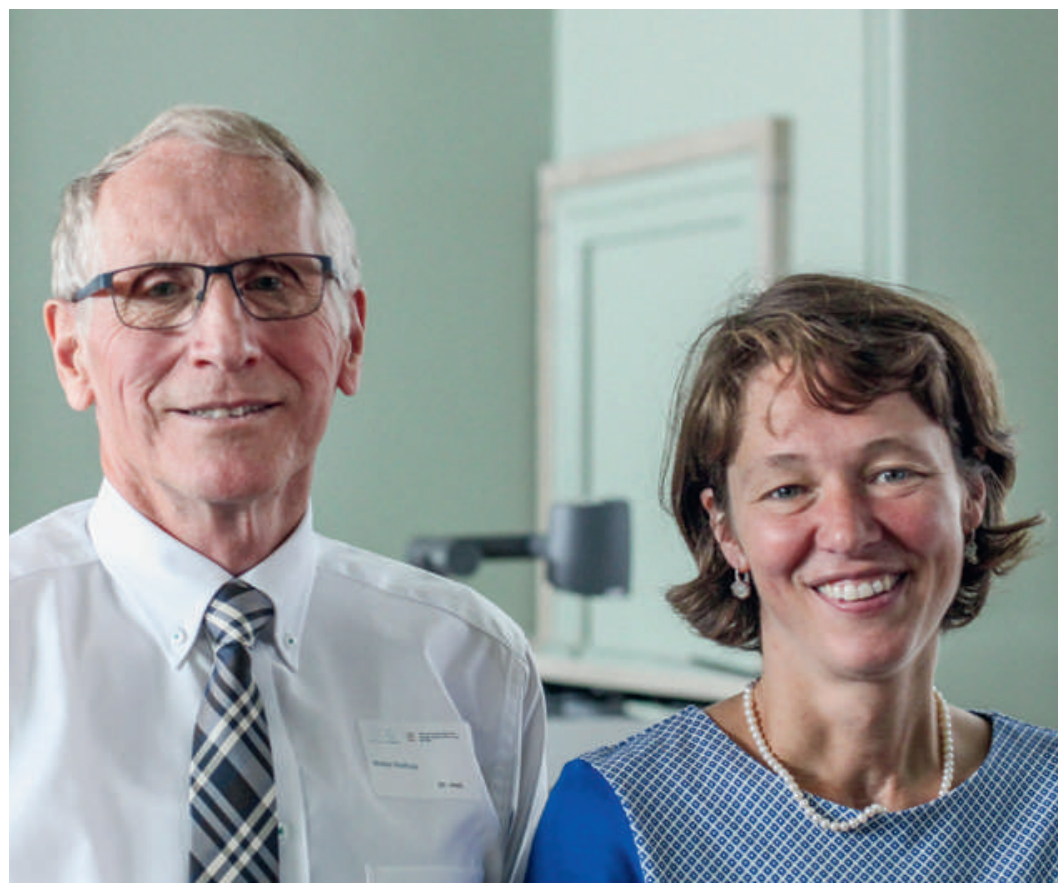

«Ermöglicht die Zusammenarbeit von Patienten, Angehörigen und Fachpersonen in der Onkologie eine bessere Qualität?» Um diese Frage ging es im Workshop des ehemaligen Krebspatienten Walter Raaflaub und Manuela Eicher.

sprächen sich die verschiedenen Empfehlungen bisweilen stark - etwa beim Thema Brustkrebs-Screening. «Weniger ist mehr», plädierte Stalder für mehr Augenmass bei den Qualitätsaktivitäten und bezog sich dabei auch auf eine entsprechende Aussage in der "Qualitätsstrategie des Bundes im Schweizerischen Gesundheitswesen» (2009): «Das Nebeneinander von Qualitätsinstitutionen und -projekten generiert gigantische Kosten und führt bestenfalls zu partiellen Erfolgen [...] der Qualitätssicherung und Patientensicherheit.»

«Wie geht es heute Ihrer Fähigkeit, mit der Krankheit zurechtzukommen?»

Auch die Befragung der Patienten im Sinne der Qualitätsmessung sei methodisch nicht einfach, indem sich etwa die Lebensqualität trotz schlechter Behandlungsaussichten erhöhen könne - Stichwort «Coping». Deshalb bevorzuge er statt der Frage «Wie geht es Ihnen heute?» die Frage: «Wie geht es heute Ihrer Fähigkeit, mit der Krankheit zurechtzukommen?»

\section{Strategie gegen Krebs}

In Bezug auf Krebs bedeutet das Coping für die Betroffenen immer öfter, sich auf eine chronische Krankheit einzustellen. Dank Fortschritten in Diagnostik, Behandlung und Nachsorge steigt die Lebenserwartung von Krebskranken. Gleichzeitig lässt die demographische Entwicklung für die kommenden Jahre eine weitere Zunahme der Krebserkrankungen in der Schweiz erwarten.

Nicht zuletzt vor diesem Hintergrund hat der Bund die Nationale Strategie gegen Krebs 2014-2017 (NSK) entwickelt, wozu am Kongress der Stand der Arbeiten präsentiert wurde. Die in der NSK vorgesehenen Projekte reichen von der Definition von Patientenpfaden und dem Ausbau von Beratungsangeboten für Patienten bis zur Förderung der klinischen Krebsforschung.

Die Strategie sieht zudem vor, dass die Schweiz ein Krebsregistrierungsgesetz erhält. Das Gesetz soll die Erhebung, Registrierung und Weiterleitung von Daten zu Krebserkrankungen auf nationaler Ebene regeln. Es sieht die Einführung einer Meldepflicht von diagnostizierten Erkrankungen vor, wobei die Patientinnen und Patienten der Registrierung ihrer Daten widersprechen können. Der Bundesrat hat den Entwurf zum Gesetz im Oktober 2014 ans Parlament überwiesen, das die Beratung dazu im Frühling begonnen hat.

Dass Daten zu Krebserkrankungen und deren Verlauf wertvolle Hinweise geben können auf den Erfolg von Behandlungen und darum wichtig sind im Rahmen der Qualitätsdiskussion, darüber waren sich die Teilnehmer des 2. Schweizer Krebskongresses einig. Solche Register würden auch für andere chronische Erkrankungen Sinn machen, meinte ein Teilnehmer. Das geplante Krebsregistrierungsgesetz sieht vor, dass der Bund Register $\mathrm{zu}$ anderen stark verbreiteten oder bösartigen nicht-übertragbaren Krankheiten finanziell unterstützen kann.

\section{Zusammenarbeit verstärken}

Mit welchen anderen Massnahmen lässt sich eine möglichst hohe Qualität in der Prävention, Behandlung und Nachsorge bei Krebserkrankungen erreichen? In zehn Workshops beschäftigten sich die Kongress-Teilnehmenden vertieft mit dieser Frage. Exemplarisch seien im Folgenden einige Aspekte näher beleuchtet.

Gerade weil Krebs immer öfter eine chronische Krankheit sei, so wurde am Kongress mehrfach gesagt, werde die interdisziplinäre Zusammenarbeit wichtiger - von den ärztlichen und pflegerischen Fachpersonen der Onkologie und Chirurgie bis zur Onko-Rehabilitation und Palliativmedizin.

Qualitätskriterien in der Krebsbehandlung müssten gemeinsam mit den Patienten formuliert werden, forderte ein Workshop-Teilnehmer. Auch die Charta «Zusammenarbeit der Fachleute im Gesundheitswesen» der Schweizerischen Akademie der Medizini- 


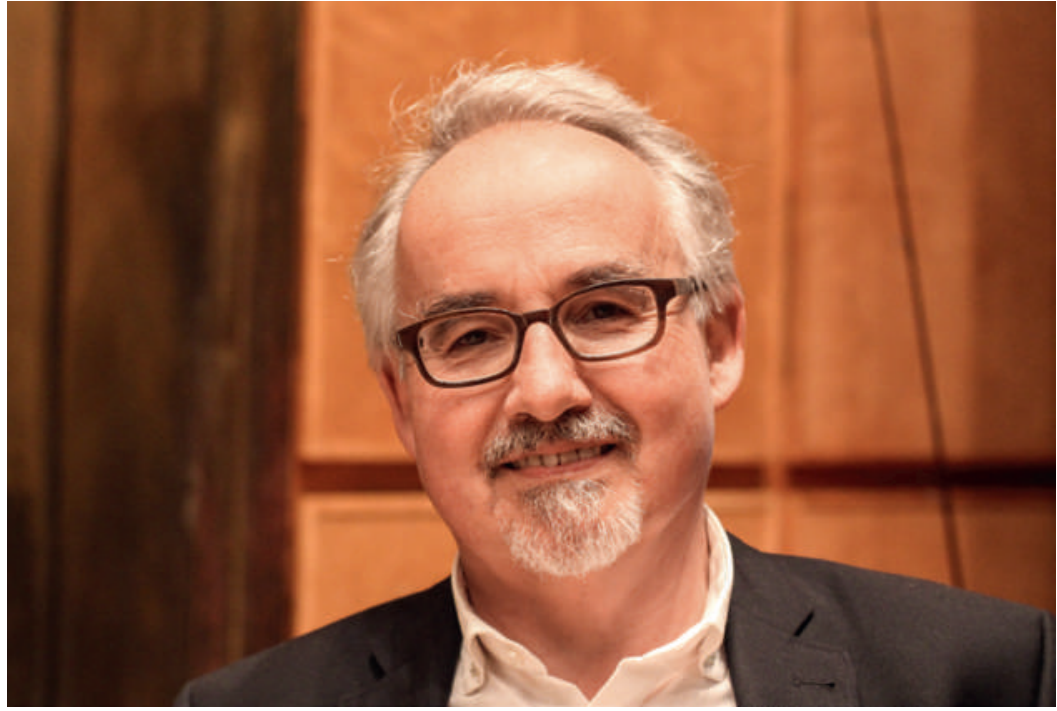

Wie sollen angesichts zunehmender und chronisch verlaufender Krebserkrankungen die finanziellen Ressourcen eingesetzt werden? Thomas Cerny fordert eine politische Debatte.

schen Wissenshaften (SAMW) empfiehlt, die Patientinnen und Patienten als «Experten in eigener Sache» in die interprofessionelle Zusammenarbeit einzubinden.

Manuela Eicher, von der Abteilung für angewandte Forschung und Entwicklung der Hochschule für Gesundheit Fribourg, regte an, die Perspektive der Patienten und Angehörigen in Forschung und Praxis stärker zu berücksichtigen. Sie erläuterte am Beispiel

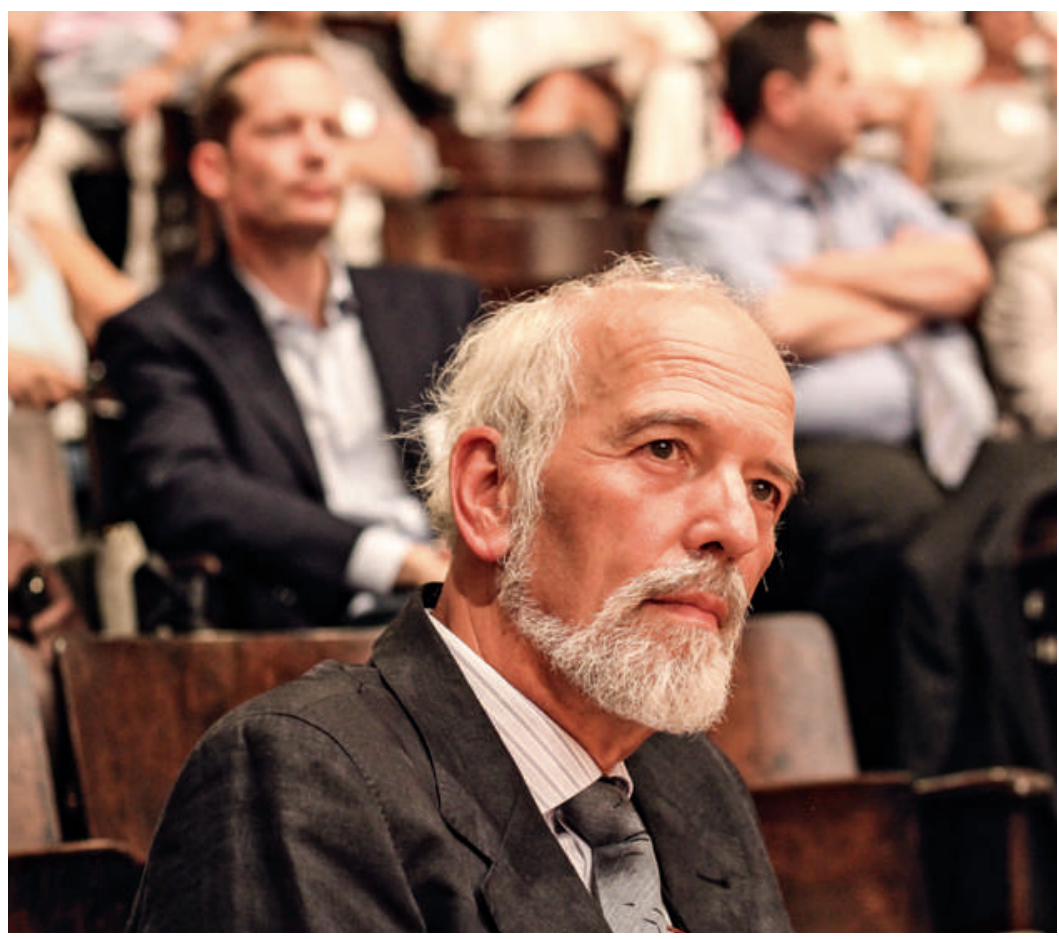

Jürg Nadig, Präsident der Schweizerischen Gesellschaft für Medizinische Onkologie, folgt den Referaten am 2. Schweizerischen Krebskongress. einer kürzlich publizierten Studie, dass Ärztinnen und Ärzte bei neu getesteten Krebsmedikamenten deutlich weniger Nebenwirkungen zurückmelden als die Patienten. Die Stimme der Patienten mittels sogenannter «Patient Reported Outcomes» direkt zu hören, sei deshalb wichtig.

Ein nachahmenswertes Beispiel ist für Eicher das British Medical Journal. Sogenannte "Patient Reviewer» werden dort vor der Publikation in den üblichen Reviewprozess von Studien einbezogen. Damit will die Zeitschrift die Relevanz und Patientenzentriertheit der Publikation von Forschungsergebnissen verbessern.

\section{Arbeit an der Kultur}

Mehrfach diskutiert wurde am Kongress, ob es nicht "Lotsen» brauche, welche die Krebspatienten durch den Behandlungsprozess begleiten. In den USA und Kanada übernehmen "nurse navigators» oder "patient navigators" vielerorts diese Aufgabe. In der Schweiz übernehmen Pflegende als «Breast Care Nurses» bei Brustkrebspatientinnen heute solche Rollen. Allerdings

\section{Die Perspektive von Patienten}

und Angehörigen sollte in Forschung und

Praxis stärker berücksichtigt werden.

können deren Leistungen gemäss Manuela Eicher derzeit nicht ausreichend abgerechnet werden, da es an entsprechenden Positionen etwa im TARMED fehlt. Zumindest bei komplexen Fällen wäre eine solche Begleitung sinnvoll, wurde im Workshop gesagt.

Besonders brisant werden Fragen der Qualität, wenn die Sicherheit der Patienten nicht mehr gewährleistet ist. David Schwappach, wissenschaftlicher Leiter der Stiftung "Patientensicherheit Schweiz», berichtete von einer Studie [1], in der er Ärzte und Pflegefachpersonen von neun onkologischen Abteilungen in der Schweiz befragt hatte. Das bedenkliche Resultat: 72 Prozent der Befragten hatten in den vergangenen 12 Monaten Bedenken zur Patientensicherheit im Team nicht geäussert und 38 Prozent hatten im selben Zeitraum mindestens einmal geschwiegen, obwohl ihre Hinweise möglicherweise eine Gefahr für den Patienten reduziert hätten.

Wichtig sei deshalb die Arbeit an der Kommunikationskultur im Team, waren sich die Workshop-Teilnehmer einig. "Bei uns hat vom medizinisch-technischen Assistenten bis zum Professor jede Person im Team das Recht, Stopp zu sagen, wenn er oder sie ein ungutes Gefühl hat», berichtete eine Teilnehmerin. Ein gutes Teambewusstsein mit klarer Rollenteilung und guter Kommunikationskultur, standardisierte Abläufe 


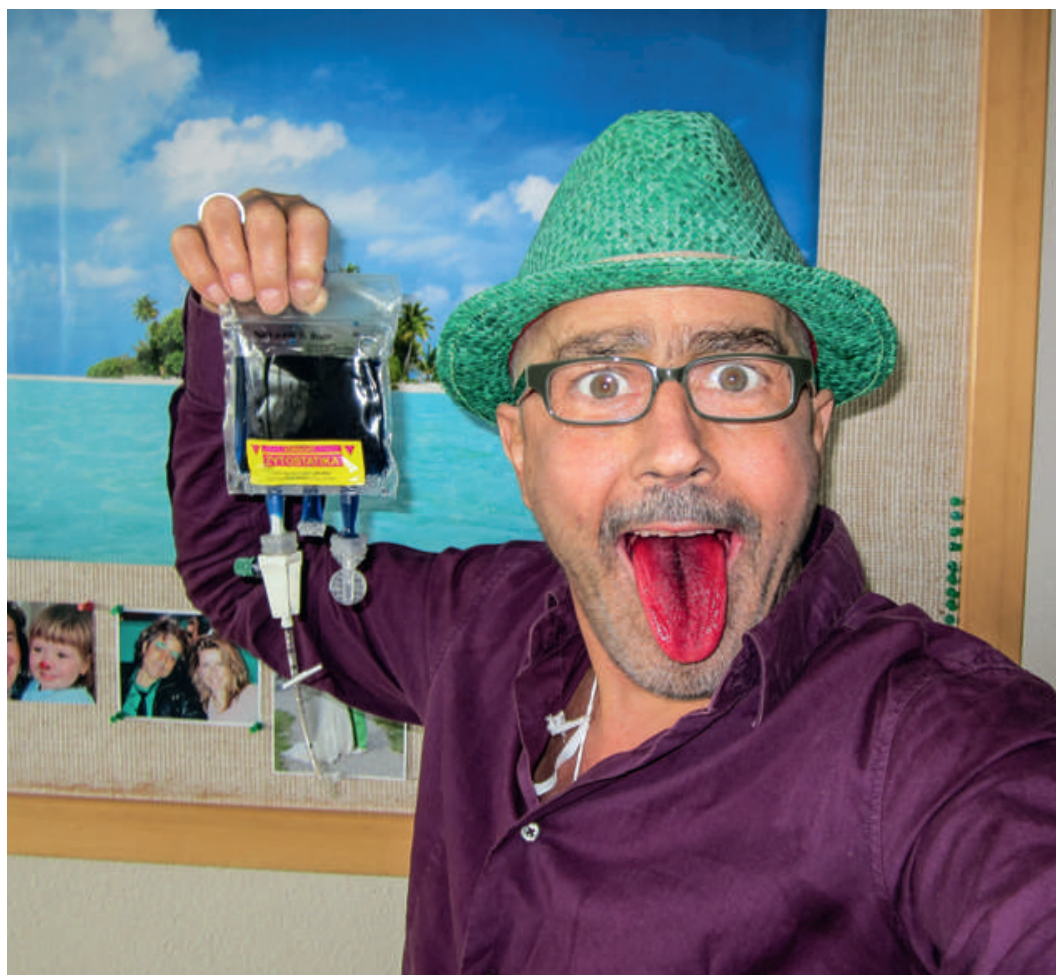

Clown «Baldrian» gelang es, mit Humor seiner Leukämie-Erkrankung etwas von ihrem Schrecken zu nehmen. Einmal nahm er die Ärzte mit seiner roten Zunge auf den Arm.

und ein optimaler Einsatz von Technologie zur Verhinderung von Fehlern fördern die Qualität, lautete die Rückmeldung des Workshops ans Plenum.

\section{Über Ressourcen reden}

Professor Thomas Cerny, Chefarzt Onkologie am Kantonsspital St. Gallen und Vorstandsmitglied der Krebsliga Schweiz, forderte eine politische Debatte darüber, wie angesichts zunehmender und zunehmend chro-

Wichtig sei die Arbeit an der Kommunikationskultur im Team.

nisch verlaufender Krebserkrankungen die finanziellen Ressourcen im Gesundheitswesen einzusetzen seien. Auf die medizinische Behandlung bezogen, forderte er ein Überdenken der Aufgabenverteilung: «Die Entwicklung wird dahin gehen, dass Pflegende vermehrt entsprechend ihrer Ausbildung gewisse weitere Tätigkeiten übernehmen - etwa selbständige Kontrolluntersuchungen und die Überwachung von Therapien auch im ambulanten Bereich.»

Was das Verhältnis von Arzt und Patient anbelangt, so plädierte Jürg Nadig, Präsident der Schweizerischen Gesellschaft für Medizinische Onkologie (SGMO), für zwei Pfeiler bei der Entscheidung für eine Behandlung: «Evidence-based» sollte sie sein und als «Shared Decision Making» zustande kommen.

Ein zentraler Wert angesichts der schweren Erkrankung ist dabei auch die Empathie gegenüber den Patientinnen und Patienten, wie der Allgemeinmediziner und ehemalige Krebspatient Walter Raaflaub in einem Workshop betont hatte: «Nur so kann Vertrauen in die Behandlung entstehen.»

\section{Rote Zunge für die Ärzte}

Vom gelungenen Aufbau dieses Vertrauensverhältnisses berichtete zum Schluss des Kongresses Thomas Leuenberger alias Clown «Baldrian». Er erzählte von seiner Leukämie-Erkrankung im Jahre 2012, bei der es ihm immer wieder gelang, mit Humor der Krankheit etwas von ihrem Schrecken zu nehmen. Manchmal unterhielt - oder schockierte - er damit auch das Behandlungsteam. So brachte ihm seine Frau etwa Randensaft ins Spital. "Als ich merkte, wie dieser die Zunge knallrot färbt, kam mir die Idee, eine neue Nebenwirkung einer Chemotherapie vorzutäuschen. Die Ärzte haben mich bei der Visite ernst genommen. Lachend habe ich sie dann über den Randensaft aufgeklärt. So hatte ich für einen Moment die Bühne im Bett und viele lachende Gesichter um mich herum.» Auch wenn es tragischere Geschichten als die seine gebe, so sei es doch wichtig, seine Ressourcen einzusetzen - in seinem Fall eben den Humor - und sich nicht aufzugeben, sagte Leuenberger. Als er über den Tod nachdachte, wurde ihm klar: "Mein bisheriges Leben war sehr gut. Was jetzt noch kommt, ist Zugabe.»

Bildnachweise

Clown: zVg.; restliche Bilder: Adrian Ritter 